\title{
An all-in-one supercapacitor working at sub-zero temperatures
}

\author{
Yanfang Wang ${ }^{1}$, Huimin Yuan ${ }^{1}$, Youhuan $\mathrm{Zhu}^{1}$, Zhiqiang Wang ${ }^{1}$, Ziwei Hu ${ }^{1}$, Jiwei Xie ${ }^{1}$, \\ Chengzhu Liao ${ }^{1}$, Hua Cheng ${ }^{1^{*}}$, Fucai Zhang ${ }^{2^{*}}$ and Zhouguang $\mathrm{Lu}^{{ }^{*}}$
}

Among energy storage devices, electrochemical capacitors (ECs) possessing ultrahigh power density and long-term stability have attracted numerous research interests and thus enriched our arsenal when coping with specific issues [1-10]. While a large number of aqueous ECs could run well at room temperature, demand for their survival and operation under thermal extremes is rising. For example, military-grade electrochemical energy storage (EES) devices are expected to operate from -40 to $60^{\circ} \mathrm{C}$ and equipment for cold climates or high altitudes can experience temperatures as low as $-60^{\circ} \mathrm{C}$ [11]. However, as temperature goes lower to sub-zero region, aqueous electrolytes suffering from the formation of ice and consequent precipitation of electrolytic salt usually show rapid decrease of ionic conductivity, which in turn hampers aqueous ECs from delivering desirable performances. Thus, developing anti-freezing aqueous electrolyte capable of working at extremely low temperatures is of great importance, and fortunately there are many lessons we can learn from the nature. For example, in the Arctic Circle, the deep ocean water under glaciers exists in the liquid state because of its high salinity. Also, functional chemicals such as antifreeze proteins endow some living species with the ability of surviving in polar circles. Actually, on the basis of Raoult's law, the freezing point of a non-volatile solution could be calculated according to the equation:

$T_{\mathrm{f}}=T_{\mathrm{f}}^{*}-K_{\mathrm{f}} \cdot b(B)$,

where $T_{\mathrm{f}}^{*}$ is the freezing point of solvent $(\mathrm{K}), K_{\mathrm{f}}$ is the freezing point constant $\left(\mathrm{K} \mathrm{kg} \mathrm{mol}^{-1}\right)$, and $b(B)$ refers to the molality of solute $\left(\mathrm{mol} \mathrm{kg}^{-1}\right)$. Since $T_{\mathrm{f}}^{*}$ and $K_{\mathrm{f}}$ are constants determined by the solvent, the freezing point of a specific solution decreases with increasing concentra- tion of solute. For instance, the concentrated aqueous solutions have been reported for supercapacitors working at temperatures below $0^{\circ} \mathrm{C}$ due to their lower freezing points [12-14].

For aqueous electrolytes, another approach to resist low temperatures is replacing pure water with binary solvents, a strategy widely applied in cryoprotectants. For example, as a well-known anti-freezing agent, ethylene glycol (EG) could form strong $\mathrm{H}$-bonds with water molecules and prevent the formation of crystal ices. Thus, electrolytes using water/EG mixture as solvent have been proved to be reliable for ECs at temperatures as low as $-30^{\circ} \mathrm{C}[15,16]$. Recently, the anti-freezing binary solvent system has also been successfully employed in fabricating conductive organohydrogels with polyvinyl alcohol (PVA), which exhibit stable flexibility and strain-sensitivity at temperature of $-40^{\circ} \mathrm{C}$ and thus pave the way for their application in flexible devices [17].

Herein, we propose an all-in-one supercapacitor comprising multiwall carbon nanotubes (MWCNTs) electrodes and PVA-KOH organohydrogel electrolyte. In the organohydrogel electrolyte, EG and water interact with hydrophilic hydroxyl groups in PVA and induce the gelation through a simple cooling-thaw process, while $\mathrm{KOH}$ acting as ion carrier is well-dispersed within the crosslinking network. And then by infusing the above electrolyte into the 3D MWCNTs network, electrode-electrolyte interfaces stable at sub-zero temperatures are obtained. As a consequence, the all-in-one supercapacitor shows ideal capacitive behavior at temperatures as low as $-36^{\circ} \mathrm{C}$ and excellent cycling performance with no capacitance decay after 10,000 cycles at $-18^{\circ} \mathrm{C}$. This work will shed light on further fabrication of anti-freezing energy storage and conversion devices.

\footnotetext{
${ }^{1}$ Department of Materials Science and Engineering, Southern University of Science and Technology, Shenzhen 518055, China

${ }^{2}$ Department of Electronic and Electrical Engineering, Southern University of Science and Technology, Shenzhen 518055, China

* Corresponding authors (emails: chengh@sustech.edu.cn (Cheng H); zhangfc@sustech.edu.cn (Zhang F); luzg@sustech.edu.cn (Lu Z))
} 
Fig. 1 schematically illustrates the procedure used herein to fabricate the all-in-one supercapacitor (details are available in the Supplementary information). Firstly, the MWCNTs were modified with surface functional groups via a common acid-treating method. Taking advantage of the interaction between the surfactant (cetyltrimethyl ammonium bromide, CTAB) and functional groups, the surface-modified MWCNTs were re-dispersed in deionized (DI) water, forming a stable colloidal solution. Then, the MWCNTs films were prepared by vacuum filtration of the dispersion onto a cellulose membrane along with washing by excessive DI water to remove the surfactant. The films were dried overnight to evaporate the water and infused by gel electrolyte to produce flexible electrodes. Generally, a flexible supercapacitor is comprised of a gel electrolyte sandwiched by two electrodes. Meanwhile, in most cases, the gel electrolyte only contacts the surface of electrodes or partially infills the porous electrodes, resulting in insufficient formation of electrode-electrolyte interfaces (Fig. S1). Under those conditions, additional liquid electrolytes are needed to provide extra pathways for ions and activate the system to reach its full potential. As a result, such a system often suffers from poor cycling stability due to the inevitable loss of solvent in long-term operation. In this work, with a bottom-up infilling method and a consequent cooling-thaw process, the porous MWCNTs films were fully infilled with PVA-KOH organohydrogel electrolyte, maximizing the formation of electrode-electrolyte interfaces and making them ideal for flexible supercapacitors. Shortly, the electrolyte with dissolved $\mathrm{KOH}$ was cast into dried MWCNTs films in which cellulose membranes acted as gas-permeable substrates. A waterimpermeable cover was then placed over the organosol, forcing the solvents in the electrolyte to evaporate downwards and taping into the capillary forces to continuously refill the pores [18]. On the other hand, in the binary solvent system, the strong hydrophilic hydroxyl groups on the PVA chains endow them with good affinities to EG and water molecules, thereby inducing facile gelation through H-bonding (Fig. 2a) [19]. Consequently, the gel electrolyte shows a freezing point lower than $-40^{\circ} \mathrm{C}$ and maintains good ionic conductivities at subzero temperatures (Fig. $2 \mathrm{~b}$ and Fig. S2). Besides, the cooling process facilitates the crosslinking and renders the PVA film certain degree of stretchability (Fig. 2c). After being dried, the gel-infused MWCNTs films can be detached and inherit the flexibility of PVA films (Fig. 3a). Via layer-by-layer stacking, two MWCNTs films were separated by a PVA film and pressed to form a symmetric supercapacitor. A field emission scanning electron microscope (FESEM) was applied to examine the morphologies of the as-fabricated supercapacitor. As shown in Fig. 3b-d, the front side of MWCNTs electrode contacting with the gas-permeable substrate shows rough surfaces with bare MWCNTs, rendering it good connection with current collectors. The sandwich-like structure could be clearly seen from the cross-sectional SEM image (Fig. 3e). Besides, the energy-dispersive X-ray spectroscopy (EDS) mapping analyses of the cross section reveal well spatial distribution of $\mathrm{O}, \mathrm{K}$ and $\mathrm{C}$ (Fig. 3f).

To better evaluate the gel distribution within porous electrodes, thicker MWCNTs films were also prepared and separated by a PVA film in a reversed configuration, i.e., the front side with bare MWCNTs contacts with the PVA film directly. As shown in Fig. S3a, b, in the gelinfused MWCNTs films, the rugged surface with some vertically standing MWCNTs is well-maintained, favoring fast electron transportation between electrode materials and current collectors. There is an interface due to

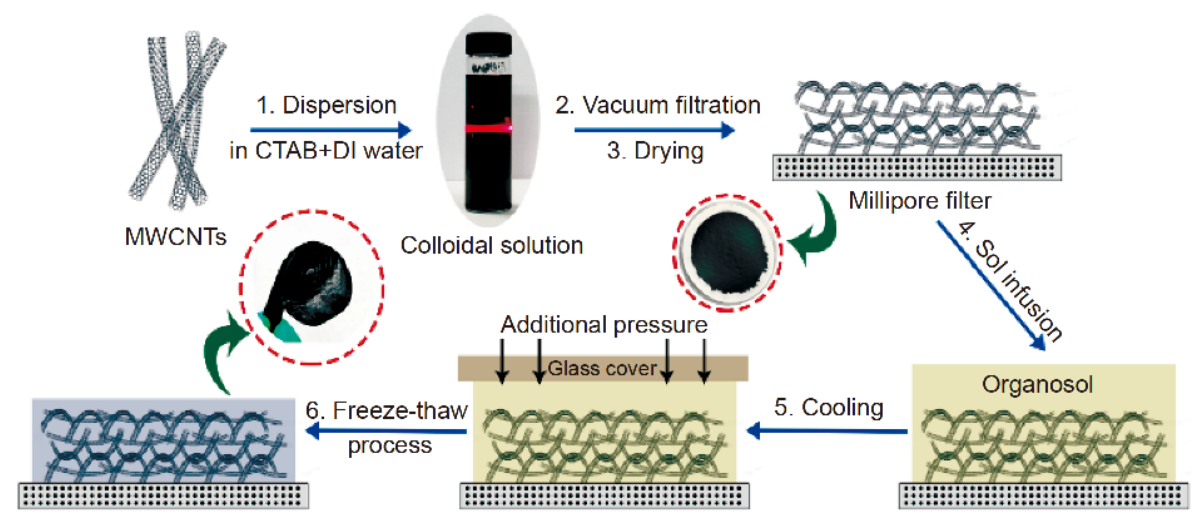

Figure 1 Schematic illustrating the preparation procedure of MWCNTs films and the corresponding gel-infused MWCNTs electrodes. Millipore filter refers to the porous cellulose membrane, and organosol refers to viscous PVA- $\mathrm{KOH}$ electrolyte dissolved in EG/ $\mathrm{H}_{2} \mathrm{O}$ binary solvent. 

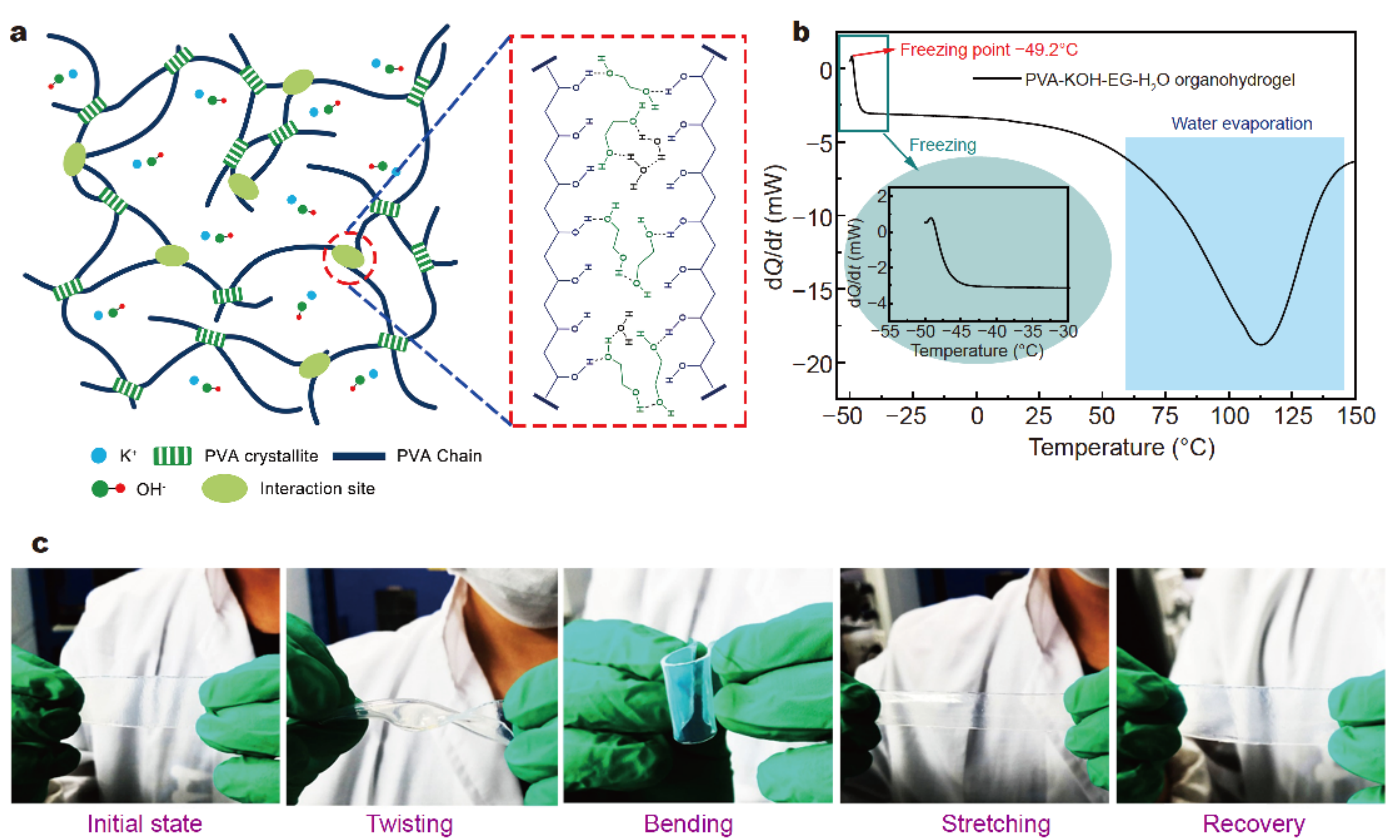

Bending

Stretching

Recovery

Figure 2 (a) Schematic illustration, (b) DSC curves and (c) optical images of the PVA-KOH organohydrogel.
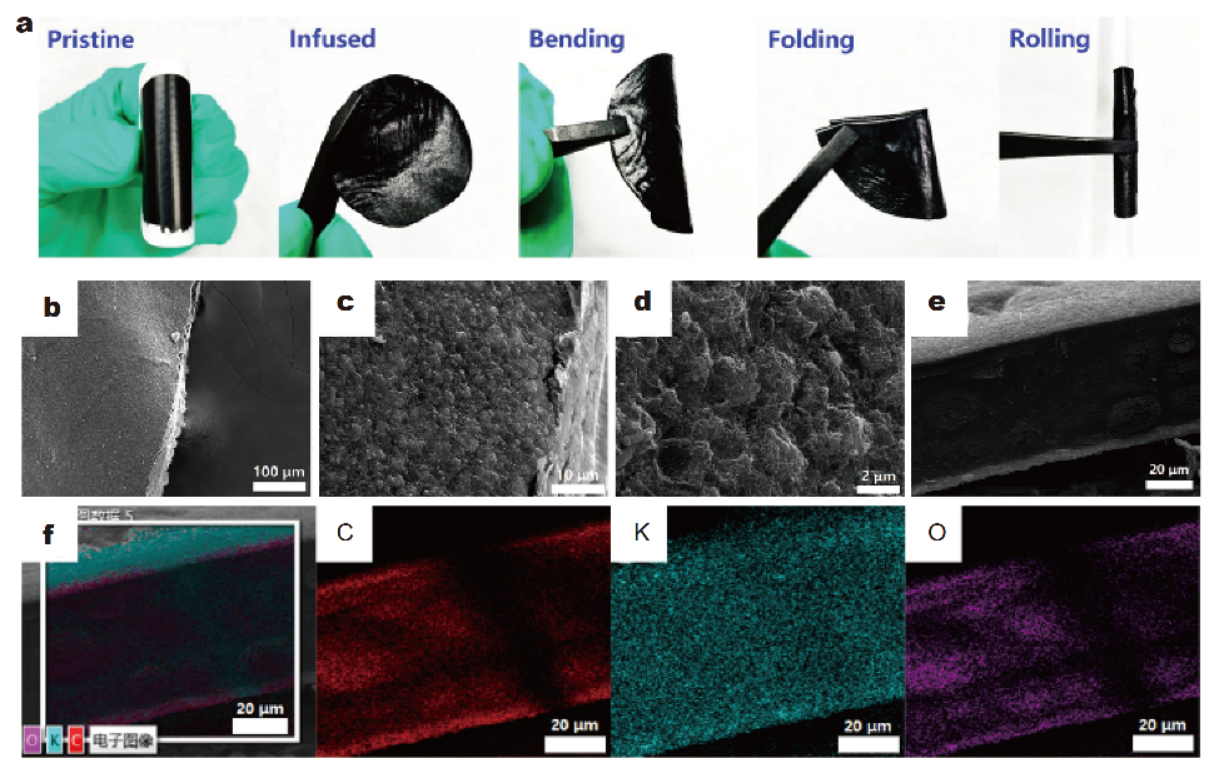

Figure 3 (a) Optic images of MWCNTs films and gel-infused MWCNTs electrodes. SEM images of the all-in-one supercapacitor from top view (b-d) and cross-sectional view (e). (f) EDS mapping images of the all-in-one supercapacitor, showing the distribution of $\mathrm{C}, \mathrm{K}$ and $\mathrm{O}$.

the extra amount of electrolyte, and both the enlarged SEM image and the EDS mapping results demonstrate the uniform distribution of gel electrolyte in the MWCNTs network (Fig. S3c, d).

After being assembled in a coin cell, electrochemical behaviors of the all-in-one MWCNTs electrode were evaluated. Fig. S4 shows its CV curves over the tem- perature range of room temperature $\left(\mathrm{RT}, 25^{\circ} \mathrm{C}\right)$ to $-36^{\circ} \mathrm{C}$. Clearly, at all temperatures, CV curves present rectangular shapes which maintain well with increasing scan rates from 2 to $20 \mathrm{mV} \mathrm{s}^{-1}$, revealing its good capacitive behavior [20]. Also, such a good capacitive behavior does not degrade with decreasing temperatures. As shown in Fig. 4a, in several cooling cycles, rectangular CV curves 

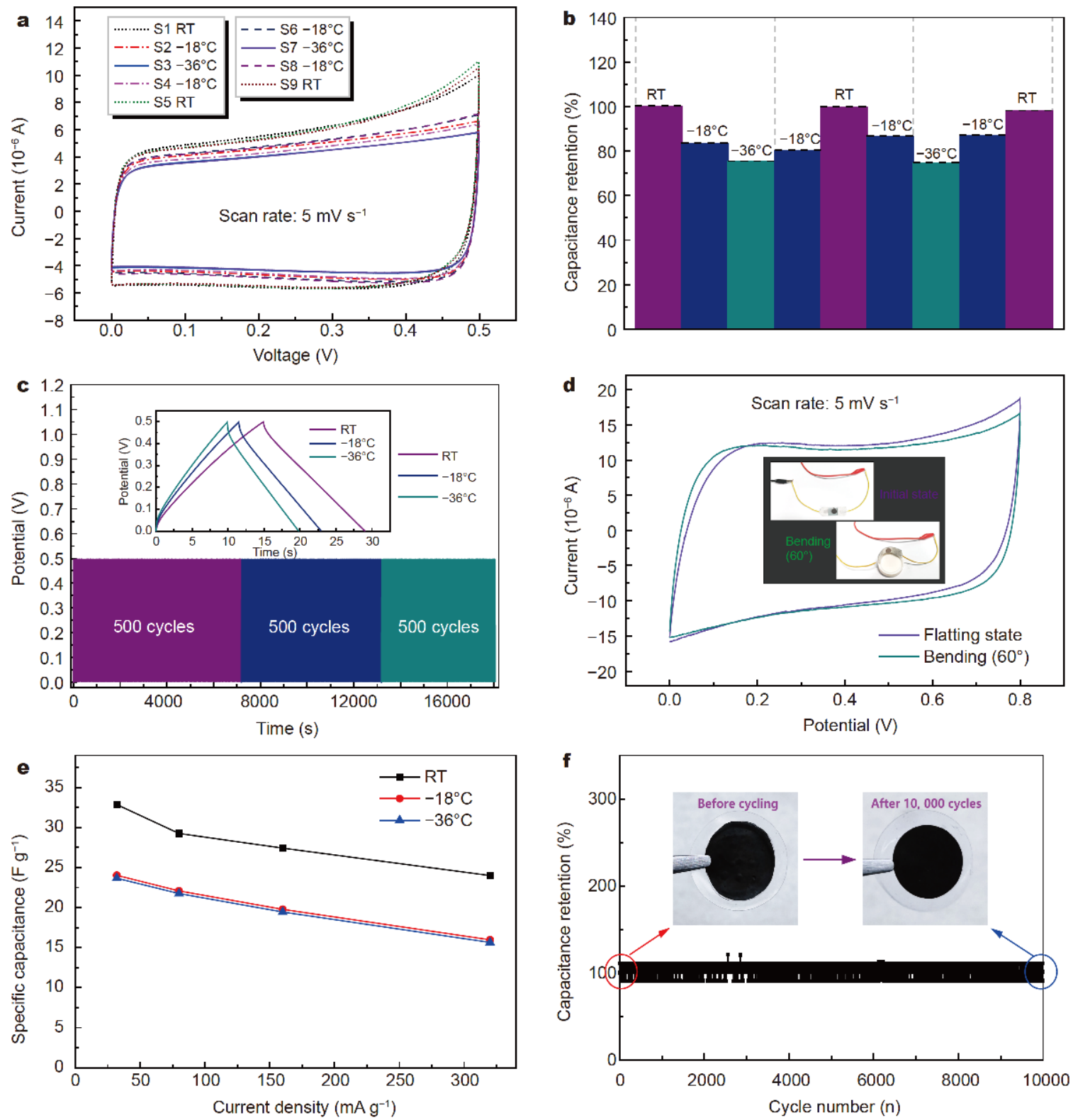

Figure 4 Electrochemical performances of the all-in-one supercapacitor. (a) CV curves and (b) the corresponding capacitance retentions over several cooling cycles. (c) GCD curves at various temperatures. (d) CV curves of flexible EC in flatting and bending states. The inset shows optic images of the flexible device. (e) Capacitances at various current densities and different temperatures. (f) Cycling performance at $-18^{\circ} \mathrm{C}$, inset: optical images of the supercapacitor before and after 10,000 cycles.

show no obvious distortion except for the shrinkage of integral areas when temperature goes lower. According to the following equation:

$C_{\mathrm{a}}=\frac{1}{v V} \int_{0}^{0.5} I_{\mathrm{a}} \mathrm{d} V$,

where $v$ is the potential scan rate $\left(\mathrm{mV} \mathrm{s}^{-1}\right), V$ is the potential $(\mathrm{V})$ and $I_{\mathrm{a}}$ is the current density $\left(\mathrm{A} \mathrm{cm}^{-2}\right)$. The EC exhibits capacitance retentions of $86.5 \%$ and $75.2 \%$ as temperature decreases from RT to -18 and $-36^{\circ} \mathrm{C}$, re- spectively. Also, the capacitance of EC experiencing several cooling cycles could return to its initial state along with temperature recovery, demonstrating its resistance to harsh conditions as well as adaptability to temperature variations (Fig. 4b). Besides, as displayed in Fig. 4c, its symmetric galvanostatic charge-discharge (GCD) curves are well-maintained as temperature decreases, indicating its superior ability of working at extreme temperatures. In details, according to the equation: 
$C_{a}=\frac{I_{a} t}{V}$,

where $t$ is the discharge time (s), such an all-in-one device shows capacitances of $1.41,1.14$, and $0.99 \mathrm{mF} \mathrm{cm}^{-2}$ at RT, -18 and $-36^{\circ} \mathrm{C}$, respectively $\left(0.05 \mathrm{~mA} \mathrm{~cm}^{-2}\right)$.

The all-in-one supercapacitor was also embedded between two indium-tin oxide coated polyethylene terephthalate (ITO-PET) films to form a flexible device and its stable potential window was measured to be $0-0.8 \mathrm{~V}$ (Fig. S5). Its CV curves are almost the same at flatting and bending states, demonstrating its workability (Fig. 4d). Fig. S6 displays its GCD performances. Not surprisingly, its capacitance decreases with increasing current densities, resulting from the larger $i-R$ drops and kinetically hampered ion transfers. As shown in Fig. 4e, the capacitances are $32.9,24.0$ and $23.7 \mathrm{~F} \mathrm{~g}^{-1}$ at $25,-18$ and $-36^{\circ} \mathrm{C}$, respectively (at $32 \mathrm{~mA} \mathrm{~g}^{-1}$ ). According to the Ragone plots (Fig. S7), its specific energy decreases from 10.5 to $7.6 \mathrm{~W} \mathrm{~h} \mathrm{~kg}^{-1}$ when the temperature goes lower from RT to $-36^{\circ} \mathrm{C}$, corresponding to $72.4 \%$ retention $\left(32 \mathrm{~mA} \mathrm{~g}^{-1}\right)$. On the basis of above results, when its capacitance decays as temperature goes lower, it could always store and release energy following ideal capacitive reactions which might be endowed by its stable configuration. If that is the case, either the decreasing response current in $\mathrm{CV}$ curves or the increasing $i-R$ drop in GCD curves should be attributed to the increasing resistance of electrolyte rather than structural damages. More direct evidences interpreting changes of ion transportation in cooling processes could be obtained from the electrochemical impedance spectroscopy (EIS) results (Fig. S8). Generally, the internal resistance of a device (notated as $R_{\Omega}$ ), i.e., the point intersecting with real axis at high frequency, includes ionic resistance of electrolyte $\left(R_{\mathrm{A}}\right)$, intrinsic resistance of electrode materials $\left(R_{\mathrm{B}}\right)$ and contact resistance $\left(R_{\mathrm{C}}\right)$ [21]. In the all-in-one $\mathrm{EC}$, as temperature decreases, the change of $R_{\mathrm{B}}+R_{\mathrm{C}}$ is negligible due to the high conductivities of MWCNTs and current collectors. Therefore, the increasement of $R_{\Omega}$ could be mainly ascribed to the sluggish ion transfer in the gel electrolyte under low temperatures, which in turn results in capacitance decline. On the other hand, the polarization resistance $\left(R_{\mathrm{P}}\right.$, i.e., the diameter of semicircle) and diffusion resistance $\left(R_{\mathrm{D}}\right.$, i.e., the length of the $45^{\circ}$ line in the middle frequency region) are often used to evaluate the penetrating ability of electrolyte into porous electrode and the migrating rate of ions from electrolyte inside pores to the surface of electrode, respectively [22]. From this perspective, the disappearance of semicircle reveals that the porous 3D MWCNTs network is fully infused with gel electrolyte, forming stable electrode-electrolyte interfaces favoring fast ion exchanges. Besides, at all temperatures, its Nyquist plots show no obvious difference in middle and low frequency regions, which further demonstrates its stable structure being able to resist cooling shocks.

In consideration of practical applications, to achieve a long lifespan is the prerequisite for any kind of energy storage device. For supercapacitors, there are typically two causes responsible for capacitance fading in longterm operation. One is that the electrode materials might suffer from physical or chemical damages, which are common phenomena in pseudocapacitors or hybrid capacitors because most of those materials store and release energy through chemical reactions $[23,24]$. In this case, many factors such as the aggregation or amorphization of electrode materials could lead to irreversible capacitance loss. The other is that from the view of devices, a specific supercapacitor might experience structural failures in long-term cycling, e.g., electrode materials shedding off from current collectors or disconnecting with electrolyte. As displayed in Fig. 4f, the all-in-one EC presents stable cycling performance with no capacitance decay after 10,000 cycles at $-18^{\circ} \mathrm{C}\left(0.04 \mathrm{~mA} \mathrm{~cm}^{-2}\right)$. Since MWCNTs could be assumed to be stable in operation, its superior cycling performances reveal that the all-in-one configuration is reliable at both RT and sub-zero temperatures (Fig. S9).

In summary, we demonstrated an all-in-one supercapacitor based on MWCNTs and PVA-KOH-EG- $\mathrm{H}_{2} \mathrm{O}$ gel electrolyte. The resultant device exhibited superior performances including high capacitance retention in long-term operation and anti-freezing capability of working at sub-zero temperatures. Actually, the all-in-one structure satisfies several requirements for advanced supercapacitors. First, the configuration with MWCNTs network buried in the gel electrolyte is stable with no agglomeration or collapse, which is to some extent like the reinforced concrete used in architecture. From this perspective, the infused gel electrolyte and the MWCNTs network function synergistically, i.e., the former renders the system certain degrees of integrity and flexibility while the later further improves its mechanical strength. Also, such an all-in-one design eliminates the usage of binders and thus evades the risk of capacitance decay induced by binder failure. On the other hand, the stable electrode-electrolyte interfaces not only allow fast charge exchanges throughout the system, but also guarantee its maintenance-free operation in a long time period. The anti-freezing feature endowed by the gel electrolyte allows it to tolerate harsh environments, and thus provides more 
opportunities of being utilized in a wide range of regions or specific fields. While using MWCNTs as electrode materials does not demonstrate its full potential because of the low capacitance, the strategy proposed herein to fabricate all-in-one supercapacitors is applicable for practical applications and further work adopting this tactic to assembly devices with higher specific capacitances without scarifying their performances is highly encouraged.

\section{Received 19 September 2019; accepted 31 December 2019; published online 14 January 2020}

1 Wang F, Wu X, Yuan X, et al. Latest advances in supercapacitors: from new electrode materials to novel device designs. Chem Soc Rev, 2017, 46: 6816-6854

2 Han P, Xu G, Han X, et al. Lithium ion capacitors in organic electrolyte system: scientific problems, material development, and key technologies. Adv Energy Mater, 2018, 8: 1801243

3 Wang Y, Chen B, Chang Z, et al. Enhancing performance of sandwich-like cobalt sulfide and carbon for quasi-solid-state hybrid electrochemical capacitors. J Mater Chem A, 2017, 5: 89818988

4 Luo H, Xiong P, Xie J, et al. Uniformly dispersed freestanding carbon nanofiber/graphene electrodes made by a scalable biological method for high-performance flexible supercapacitors. Adv Funct Mater, 2018, 28: 1803075

5 Dong $\mathrm{S}$, Chen $\mathrm{X}, \mathrm{Gu} \mathrm{L}$, et al. One dimensional $\mathrm{MnO}_{2} /$ titanium nitride nanotube coaxial arrays for high performance electrochemical capacitive energy storage. Energy Environ Sci, 2011, 4: 3502

$6 \mathrm{Hu}$ J, Li M, Lv F, et al. Heterogeneous $\mathrm{NiCo}_{2} \mathrm{O}_{4} @$ polypyrrole core/ sheath nanowire arrays on $\mathrm{Ni}$ foam for high performance supercapacitors. J Power Sources, 2015, 294: 120-127

7 Han X, Han P, Yao J, et al. Nitrogen-doped carbonized polyimide microsphere as a novel anode material for high performance lithium ion capacitors. Electrochim Acta, 2016, 196: 603-610

8 Shabangoli Y, Rahmanifar MS, El-Kady MF, et al. Thionine functionalized 3D graphene aerogel: combining simplicity and efficiency in fabrication of a metal-free redox supercapacitor. Adv Energy Mater, 2018, 8: 1802869

9 Li J, Wang N, Tian J, et al. Cross-coupled macro-mesoporous carbon network toward record high energy-power density supercapacitor at 4 V. Adv Funct Mater, 2018, 28: 1806153

10 Zhong X, Tang J, Cao L, et al. Cross-linking of polymer and ionic liquid as high-performance gel electrolyte for flexible solid-state supercapacitors. Electrochim Acta, 2017, 244: 112-118

11 Rodrigues MTF, Babu G, Gullapalli $\mathrm{H}$, et al. A materials perspective on Li-ion batteries at extreme temperatures. Nat Energy, 2017, 2: 17108

12 Su L, Gong L, Wang X, et al. Improved low-temperature performance of novel asymmetric supercapacitor with capacitor/batterycapacitor construction. Int J Energy Res, 2016, 40: 763-769

13 Su L, Gong L, Ma C, et al. Outstanding low-temperature capacitance of an AC-AC supercapacitor in acid redox electrolyte. ChemElectroChem, 2017, 4: 46-48

14 Tatlisu A, Huang Z, Chen R. High-voltage and low-temperature aqueous supercapacitor enabled by "water-in-imidazolium chlor- ide" electrolytes. ChemSusChem, 2018, 11: 3899-3904

15 Roberts AJ, Danil de Namor AF, Slade RCT. Low temperature water based electrolytes for $\mathrm{MnO}_{2} /$ carbon supercapacitors. Phys Chem Chem Phys, 2013, 15: 3518-3526

16 Vellacheri R, Al-Haddad A, Zhao H, et al. High performance supercapacitor for efficient energy storage under extreme environmental temperatures. Nano Energy, 2014, 8: 231-237

17 Rong Q, Lei W, Chen L, et al. Anti-freezing, conductive selfhealing organohydrogels with stable strain-sensitivity at subzero temperatures. Angew Chem Int Ed, 2017, 56: 14159-14163

18 Li X, Shao J, Kim SK, et al. High energy flexible supercapacitors formed via bottom-up infilling of gel electrolytes into thick porous electrodes. Nat Commun, 2018, 9: 2578

19 Sun Z, Lv F, Cao L, et al. Multistimuli-responsive, moldable supramolecular hydrogels cross-linked by ultrafast complexation of metal ions and biopolymers. Angew Chem Int Ed, 2015, 54: 79447948

20 Kale VS, Hwang M, Chang $\mathrm{H}$, et al. Microporosity-controlled synthesis of heteroatom codoped carbon nanocages by wrap-bakesublime approach for flexible all-solid-state-supercapacitors. Adv Funct Mater, 2018, 28: 1803786

21 Bard A, Faulkner L. Electrochemical Methods: Fundamentals and Applications. Hoboken: Wiley, 2000

22 Wang Y, Chen B, Zhang Y, et al. ZIF-8@MWCNT-derived carbon composite as electrode of high performance for supercapacitor. Electrochim Acta, 2016, 213: 260-269

23 Liu X, Zang W, Guan C, et al. Ni-doped cobalt-cobalt nitride heterostructure arrays for high-power supercapacitors. ACS Energy Lett, 2018, 3: 2462-2469

24 Sun S, Luo J, Qian Y, et al. Metal-organic framework derived honeycomb $\mathrm{Co}_{9} \mathrm{~S}_{8} @ \mathrm{C}$ composites for high-performance supercapacitors. Adv Energy Mater, 2018, 8: 1801080

Acknowledgements This work was financially supported by the Basic Research Project of Science and Technology Innovation Commission of Shenzhen (JCYJ20170817110251498), Guangdong Special Support for Science and Technology Leading Young Scientist (2016TQ03C919), and the National Natural Science Foundation of China (21603094, 21703096, and 11775105).

Author contributions Wang Y designed and performed the experiments; Wang Y wrote the paper with support from Lu Z; all authors contributed to the general discussion.

Conflict of interest The authors declare no conflict of interest.

Supplementary information Experimental details and the supporting electrochemical results are available in the online version of the paper.

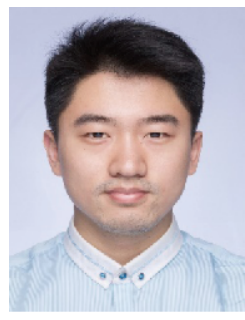

Yanfang Wang received his $\mathrm{BE}$ degree from the Central South University in 2014 and MSc degree from Fudan University in 2017. Now, he is a PhD student at Southern University of Science and Technology. His research interests mainly focus on supercapacitors and lithium-ion batteries. 


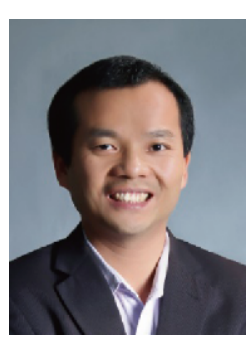

Zhouguang Lu is currently a professor in the Department of Materials Science and Engineering, Southern University of Science and Technology, China. He received his $\mathrm{PhD}$ degree from the City University of Hong Kong in 2009. He is the recipient of Fulbright Fellowship of USA Government in 2008-2009 and the Overseas High-Caliber Personnel (Level B) of Shenzhen Government in 2013. His research mainly covers the design and synthesis of nanostructures and their applications in energy storage and conversion with focus on lithium/sodium-ion and air batteries. He has authored more than 160 peer-review papers with total citations more than 5600 and $\mathrm{H}$-index of 43 .

\section{一种可在 $0^{\circ} \mathrm{C}$ 以下工作的一体式超级电容器}

王彦方 ${ }^{1}$, 袁惠敏 ${ }^{1}$, 祝友欢 ${ }^{1}$, 王志强 ${ }^{1}$, 胡紫薇 ${ }^{1}$, 谢纪伟 ${ }^{1}$, 廖成竹 ${ }^{1}$, 程化 ${ }^{1^{*}}$, 张福才 $^{2^{*}}$, 卢周广 广 $^{1^{*}}$

摘要 具有低温工作性能是超级电容器应对特殊工作环境(高海 拔/高纬度)的重要保障, 也有利于其在更多领域中的应用. 本文首 先制备了一种可在低温下保持柔韧性和离子传导性能的碱性凝胶 电解质, 并利用其制备了一种一体式超级电容器, 其可在零点温度 以下 $\left(-36^{\circ} \mathrm{C}\right)$ 仍具有稳定的工作能力. 